\title{
Filigrane
}

Écoutes psychothérapiques

\section{Le goût des mots}

\section{Marie-Ange Pongis-Khandjian}

Volume 20, numéro 1, printemps 2011

La langue vivante de la clinique psychanalytique

URI : https://id.erudit.org/iderudit/1004038ar

DOI : https://doi.org/10.7202/1004038ar

Aller au sommaire du numéro

Éditeur(s)

Revue Santé mentale au Québec

ISSN

1192-1412 (imprimé)

1911-4656 (numérique)

Découvrir la revue

\section{Citer cet article}

Pongis-Khandjian, M.-A. (2011). Le goût des mots. Filigrane, 20(1), 33-43. https://doi.org/10.7202/1004038ar

\section{Résumé de l'article}

Bien que Freud ait été polyglotte, le thème du bilinguisme et du multilinguisme n'a intéressé la psychanalyse que depuis peu. Le mythe de la tour de Babel, considéré traditionnellement comme une punition divine, peut cependant aussi être vu comme un facteur d'essor humain. L'auteur explore son enfance dans un environnement plurilingue avec ses avantages et ses difficultés. Elle interroge le lien entre la langue, la migration et l'identité culturelle. À travers de courtes vignettes, elle illustre l'utilisation de différentes langues au cours de thérapies.
Ce document est protégé par la loi sur le droit d'auteur. L'utilisation des services d’Érudit (y compris la reproduction) est assujettie à sa politique d'utilisation que vous pouvez consulter en ligne.

https://apropos.erudit.org/fr/usagers/politique-dutilisation/ 


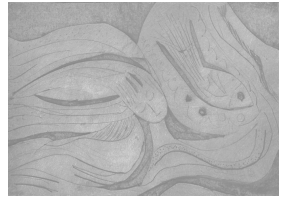

\section{Le goût des mots}

\section{Marie-Ange Pongis-Khandjian}

Bien que Freud ait été polyglotte, le thème du bilinguisme et du multilinguisme n'a intéressé la psychanalyse que depuis peu. Le mythe de la tour de Babel, considéré traditionnellement comme une punition divine, peut cependant aussi être vu comme un facteur d'essor humain. L'auteur explore son enfance dans un environnement plurilingue avec ses avantages et ses difficultés. Elle interroge le lien entre la langue, la migration et l'identité culturelle. À travers de courtes vignettes, elle illustre l'utilisation de différentes langues au cours de thérapies.

La langue est un organe d'exploration du réel, elle est un palpeur, un pseudopode perceptif et sensible avec lequel l'enfant, et plus tard l'analysant et l'analyste, explore le monde $[\ldots]$ Pour l'infans, $[\ldots]$ chaque mot entendu et qu'il voit prononcé sur les lèvres du visage aimé est aussi un objet signe qu'il incorpore dans sa bouche, qu'il savoure et déguste en imitant la prononciation, qu'il attrape et retient avec sa langue et ses lèvres, dans on souffle.

Edmundo Mango $(2009,238)$

Les vrais gourmands lisent en remuant les lèvres pour déguster les mots.

Yvan Audouard ${ }^{1}$

T ous les humains ne sont-ils pas bilingues par essence, du fait même que l'inconscient est la langue que tout le monde parle mais que personne ne comprend et tous les psychanalystes ne sont-ils pas des «écouteurs polyphoniques » et de traîtres traducteurs ${ }^{2}$ ?

«La langue analytique, découverte et inventée par Freud, est une langue étrangère, dont les entre - mots, le non - encore formulé, les transformations incessantes de sens, en font la plus vivante des langues » écrit Denise Collin-Rothberg $(1988,69)$.

Mots écrans, passagers clandestins, qui du fond de la cale, mènent la nef au grand large.

Mots muets, mots coquillages qui font entendre le bruissement de la mer(e) lorsqu'ils sont murmurés sur la pointe de l'âme et qui résonnent par leur silence plein de sens.

Mots madeleines pétris de saveurs, d'odeurs, de textures.

Mots corps traduisant les sensations infantiles devenant parfois mots cadavres, désensualisés, pris dans la gangue du rationnel, qui ne veulent plus rien dire du tout mais qui taisent surtout l'essentiel. 
Mots fossiles qui ont encrypté à jamais des trésors.

\section{Enfance multilingue}

Grandir dans un pays du Moyen-Orient implique d'entendre parler, crier, chanter en arabe, en anglais, en arménien, en français, en grec, en italien (et j'en oublie probablement), de voir au cinéma des films projetés en version originale, sous-titrée en deux langues différentes, d'être confronté à l'islam, au judaïsme et au christianisme sous ses différents subdivisions (catholiques, orthodoxes, maronites, coptes, etc.) et d'en fêter toutes les fêtes ${ }^{3}$ ! Les enfants, dans cet environnement plurilingue, apprennent sans effort un vocabulaire de base. Il est aussi habituel pour les conversations de se dérouler dans un va-et-vient entre ces différentes langues sans que personne ne s'en étonne. Privilège certes mais qui entraîne cependant son lot de difficultés. Lorsqu'on me demande quelle est ma langue maternelle, je me sens embarrassée, je n'en sais trop rien. Ma première année de vie a baigné dans une enveloppe sonore du soi (Didier Anzieu, 1987), un environnement linguistique, grec et français; il en avait été de même pour mes deux parents. Anglais et arabe s'y sont rajoutés l'année suivante. J'ai appris à parler dans ces quatre langues, très différentes les unes des autres par leur sonorité et leur structure, simultanément et sans les confondre me semble-t-il. L'apprentissage de l'écriture s'est fait, plus tard, avec trois alphabets différents (latin, grec et arabe). L'italien s'est implanté, avant l'âge de dix ans, en jouant avec de petits Italiens lors de plusieurs vacances consécutives dans les Dolomites, je l'ai beaucoup parlé, plus tard, dans la vingtaine. L'espagnol est la seule langue apprise à l'âge adulte et le processus d'apprentissage est totalement différent. L'enfant apprend par osmose, à l'oreille, de façon ludique. Il ne fait pas de traduction (du moins consciemment) d'une langue à l'autre. L'apprentissage de l'adulte est rationnel, il se soucie de grammaire, de vocabulaire, de syntaxe, etc., et la traduction est perpétuellement en arrière-plan.

Elias Canetti, écrivain d'origine bulgare, parlait le ladino (forme d'espagnol parlé au Moyen-Orient par les juifs expulsés d'Espagne au Xve siècle), le bulgare, l'anglais, le français et l'allemand. Alors qu'il n'a appris l'allemand, langue d'intimité entre ses parents, qu'à l'âge de six ans, Canetti affirme que la majorité de ses souvenirs sont gravés dans sa mémoire dans cette langue, devenue la langue d'amour entre sa mère et lui. Il parle d'une "mystérieuse transformation », "une traduction qui s'est opérée toute seule dans l'inconscient» (Canetti, 1998, 12).

À travers ces entrelacs de codes, le français s'est taillé la part du lion en moi; je pense, compte et rêve (à quelques exceptions près) en français. Alors que mon vocabulaire grec et arabe n'a pas dépassé celui de l'adolescence, anglais et italien ont atteint une certaine maturité!

Avoir à sa disposition, dans l'enfance, plusieurs mots à sonorités différentes, pour désigner un objet, un sentiment, une idée qui peuvent sembler à première vue les mêmes, mais qui prennent une autre coloration, un autre goût selon la langue utilisée, sa musique, aiguise le sens de la nuance et le goût de la différence.

Cependant, pour des personnes ayant appris sur le tard une deuxième langue le bilinguisme peut être problématique. 
Tzsvetan Todorov (cité par Amati, 1994, 66), écrivain et essayiste bulgare, émigré en France trouvait impossible de combiner ces deux mondes linguistiques. Il écrit « en changeant de langue, je me suis vu changer de destinataire imaginaire». Il a donc vécu le bilinguisme sous l'angle du clivage.

François Cheng (cité par Amati, 1994, 67), écrivain, peintre et calligraphe chinois, a été parachuté à l'âge de 20 ans à Paris, sans un mot de français. Il a aussi vécu le bilinguisme comme Todorov, «sous le signe du refoulement, du clivage et de l'exil intérieur» (cité par Amati, 1994, 68).

\section{Polyglottisme et psychanalyse}

Le langage a été objet d'intérêt pour la psychanalyse depuis ses débuts. Freud lui-même était multilingue (allemand, tchèque et yiddish). Cependant, alors que la littérature, la sociologie, l'anthropologie, la linguistique, la sémiotique se sont penchées sur la question du polyglottisme, la psychanalyse, elle, ne s'y est pas beaucoup arrêtée.

En 1932, Ferenczi a été le premier à s'arrêter à la confusion des langues entre adultes et enfant. Il ne s'agit pas d'effectuer un saut conceptuel entre langue et langage mais de souligner qu'alors que l'enfant s'exprime dans le langage de la «tendresse », les adultes lui répondent dans celui de la «passion".

Rappelons que la langue maternelle de Ferenczi était le hongrois, qu'il communiquait avec Freud en allemand et qu'écrire en anglais était peut-être pour lui un moyen de se dégager de Freud ${ }^{4}$.

Dans les années quarante plusieurs analystes de religion juive, vivant en Allemagne ou en Autriche et qui souvent parlaient plusieurs langues, émigrent en Amérique du Sud ou en Angleterre et doivent en apprendre une nouvelle. Otto Kernberg (1994, 713) émet l'hypothèse suivante: pris par leur propre tache aveugle, les analystes multilingues de cette génération n'avaient pas la distance personnelle nécessaire pour étudier les conséquences sur le transfert et le contre-transfert, des communications en des langues différentes apportées par un patient au cours d'une analyse.

Trois psychanalystes multilingues travaillant avec des patients multilingues, Jacqueline Amati Mehler, Simona Argentieri et Jorge Canestri, ont écrit un ouvrage de référence pour ceux et celles qui s'intéressent à ce sujet, La Babel de l'inconscient (Mehler et al., 1994) ${ }^{5}$. Les auteurs y explorent la différence endopsychique entre multilingues ${ }^{6}$ et polyglottes ${ }^{7}$ et se centrent sur la clarification de deux hypothèses avancées par Luisa de Urtebey $(1993,930)$ :

- Ou bien chaque langue, apprise dans l'enfance par un même sujet, fonctionne comme un vocabulaire élargi au sein d'un seul réseau de représentations de mots. Dans ce cas, par exemple, aux mots livre, book, libro, vivlio, kitab correspondrait une seule image complexe de livre.

- Ou bien chaque langue est constituée par un système de représentations de mot, clos, mais différent, organisé par ses propres lois.

Cette deuxième hypothèse me semble illustrée par l'extrait suivant, tiré du roman d'Anne Michaels (1996, 130), La mémoire en fuite. Pendant la guerre, un 
archéologue recueille un enfant juif polonais, et le cache dans l'île grecque de Zakynthos où il lui apprend l'anglais. L'enfant dit:

Je les rédigeais [les poèmes] en anglais parlé, quasi phonétiquement, mais il arrivait qu'un mot se détache des autres et prenne soudain un sens pur et net, précis. Il y avait donc une différence entre un chien grec et un chien américain, entre la neige qui tombe en Pologne et celle qui poudroie au Canada, entre les pins résineux qui poussent en Grèce et ceux qui croissent en Pologne ${ }^{8}$.

Jacqueline Amati Mehler, d'origine argentine, de religion juive, a parlé quatre langues dès l'enfance (allemand, yiddish, espagnol et français) et a appris, plus tard l'anglais et l'italien (qui est devenu sa principale langue de travail). Elle parle de sa «babelique expérience polylingue» de façon très touchante (Amati Mehler, 2003, 337).

Contrairement à ce que proclamait l'École Logopédique de Vienne, à savoir que l'exposition d'un enfant à d'autres langues que la langue maternelle cause des dégâts irréparables de la parole et des fonctions cognitives, pour Amati Mehler $(1993,923)$ «l'organisation mentale multilinguistique peut augmenter la richesse, la plasticité et le développement général du réseau symbolique».

\section{La tour de Babel: punition divine ou facteur d'essor?}

Que Babel soit un châtiment serait « un viatique de prêt-à-penser » écrit Claude Hagège (2009, 48-73), linguiste passionnément épris des langues. D’après lui, la vocation de l'humain n'est pas de se laisser prendre aux mirages de l'unique et aux tentations de repli. Y céder, c'est s'interdire le foisonnement des cultures, des langues, des types de relation avec notre environnement. Pour Hagège, toutes les langues sont menacées par la diffusion mondiale de l'anglais. Cette langue serait, selon lui «le symbole de la mort sous les trompeuses et flatteuses apparences de l'aisance à communiquer» (ibid., 169). Il rappelle qu'à partir de 1814, pendant un siècle et demi, $30 \%$ des enfants australiens autochtones furent enlevés de force à leur milieu et placés dans des familles, des orphelinats ou des internats de type carcéral où il était formellement interdit d'utiliser une autre langue que l'anglais. Au Canada les mêmes événements se sont produits. Des enfants d'autochtones furent arrachés à leur famille, placés dans des orphelinats où l'enseignement des missionnaires diabolisa leur langue maternelle. Nous pouvons nous interroger sur les dégâts découlant de cette acculturation.

Selon Hagège, quand un enfant grandit dans un milieu bilingue, il se produit une vicariance: chacune des deux langues est capable de suppléer à l'insuffisance de l'autre selon le domaine abordé; le domaine des choses de la table paraîtra, par exemple, plus naturel dans une langue alors que dans l'autre ce sera celui des choses de l'esprit. Il évoque aussi la "compétence passive», aptitude à comprendre une langue non maternelle mais liée à une parenté avec elle. Par exemple, le danois, le suédois et le norvégien sont si proches que l'habitant de chaque pays peut comprendre l'autre. Il en va de même entre l'italien, l'espagnol et le portugais. 


\section{Langue, migration et identité: "je parle donc je suis..."}

«Dans quelle mesure notre identité tient-elle à la langue que nous parlons, dans laquelle nous nous pensons? Suis-je la même personne quand je parle allemand, français ou anglais?» se demande Liselotte Marchal dans son roman Les mots étranglés (Marchal, 2000, 47).

Sa question me paraît équivalente à celle de se demander si nous sommes la même personne lorsque nous sommes rouges de colère, bleus de peur, verts de jalousie ou blancs d'anxiété. Kaléidoscope d'émotions humaines traduit par un kaléidoscope de mots. Représentations de choses transformées en représentations de mots.

Par ailleurs Hector Bianciotti ${ }^{9}$, dans une interview parue dans le journal $L e$ Monde affirme qu' «on peut être désespéré dans une langue, à peine triste dans l'autre» (cité par Denise Colin-Rothberg, 1988, 65).

Une collègue m'a soumis la réflexion suivante: la langue peut définir une partie de l'identité pour quelqu'un dont c'est un aspect déterminant (par exemple le français pour les Québécois), mais que le plurilinguisme peut définir l'identité d'une personne qui a été élevée dans plusieurs langues. Autrement dit, ce n'est pas tant la langue qui définit l'identité, mais plutôt comment chacun l'a vécue dans son enfance.

Léon et Rebecca Grinberg ${ }^{10}$, intéressés par le thème de la migration du fait de leur propre expérience migratoire, ont étudié les vicissitudes de ce processus du point de vue, entre autres, de la langue et de l'identité. Pour les Grinberg, «Le langage "crée" l'image que nous nous faisons de la réalité, et nous impose cette image en même temps ». Cela expliquerait selon eux «les énormes difficultés de l'immigrant à "changer" de langue, produit de la culture qu'il a "tétée" et qui lui a servi depuis qu'il était petit pour "créer" et assimiler l'image du monde qui l'entourait» (Grinberg, 1986, 127). La migration met à l'épreuve la stabilité psychique et émotionnelle. Elle exerce souvent une fonction déstabilisatrice sur le sentiment d'identité. Mais «dans certains cas, la migration peut être décidée de manière consciente ou inconsciente, comme une façon de tenter d'affermir le sentiment d'identité, à travers le contact plus direct avec les endroits qui constituent le berceau des ancêtres. Ces lieux, à travers les récits familiaux, les lectures et les traditions, [...] représentent les racines lointaines et désirées ${ }^{11}$ » (ibid., 167).

L'être humain est capable de continuer à se sentir lui-même à travers une succession de changements, de maintenir une stabilité intérieure malgré les transformations de sa vie. Mais quelle est la limite tolérable pour que cela ne nuise pas de façon irrémédiable à l'identité? Les Grinberg ont envisagé le sentiment d'identité comme résultat d'un processus d'interaction continuelle entre trois liens d'intégration: spatial, temporel et social (Grinberg, 1971). Ils ont pu étudier ces liens dans le contexte de la relation patient-analyste. Ils estiment que ces trois liens sont affectés d'une manière générale par la migration.

La perturbation du lien d'intégration spatial peut se manifester par des états de désorganisation, à des degrés variables. Des angoisses très primitives peuvent surgir produisant des états de panique, de dépersonnalisation ou de déréalisation (par exemple lors de crainte d'être «dévoré», «mis en pièces" par la nouvelle culture). 
D’affolantes questions - «Où suis-je », «Qu'est-ce que je fais ici ? - peuvent surgir comme au réveil, dans un état de demi-sommeil. Dans les cas extrêmes, la personne peut être sidérée comme si les différents «morceaux» de son identité ne sont plus reliés harmonieusement.

Le mélange des souvenirs avec des situations actuelles, les lapsus confondant des lieux ou des personnes du passé mettent en évidence une perturbation dans le lien temporel.

Le lien d'intégration social est, selon les Grinberg, celui qui est le plus manifestement affecté par la migration puisque les plus grands changements se produisent justement par rapport à l'environnement, où tout est nouveau et où l'émigrant est un «inconnu» pour les autres, accentuant ainsi son sentiment de «non-appartenance ».

J'ajouterai que lorsqu'il arrive à l'émigrant de retourner dans son pays d'origine, il se peut aussi qu'il y soit reçu comme un inconnu par ceux qu'il considérait comme les siens. Il n'appartient plus ni à ce bord ni à l'autre...

De retour dans le pays de mon enfance après plus de quarante ans d'absence, j'ai pu constater combien les expressions de la langue parlée peuvent changer. Pensons aussi à l'argot français (à peu près incompréhensible) des jeunes d'aujourd'hui...

La question de l'identité culturelle m’interpelle depuis longtemps. Le «Quelle est ta langue maternelle?» implique aussi un «Qui es-tu? D’où viens-tu?». Comment répondre à cette question lorsque parents et grands-parents proviennent de la dispersion d'une communauté à travers le monde (diaspora arménienne, chinoise, grecque, libanaise, etc.)?

Lorsque j'ai émigré en Europe pour étudier à l'université, alors que jusque-là la question de la «langue maternelle» ne m'avait pas effleurée, le regard interrogatif des autres pointait une sorte de problème. En Suisse, sur les quatre langues nationales (français, allemand, italien et romanche) auxquelles se rajoutent les différents dialectes (zurichois, bernois, appenzellois, bâlois), les enfants en apprennent deux. Chacun garde un accent bien distinct, mais moi je n'entrais dans aucune case...

En fait, je fais partie des «immigrants privilégiés». Mes changements de pays ont été choisis, décidés, vécus dans de bonnes conditions et dans des environnements accueillants. À aucun moment, je n'ai dû abandonner une langue, m'exiler d'elle. Je les transporte toutes en moi, un peu en dormance et, selon les circonstances, elles sortent de leur engourdissement, s'ébrouent et reprennent leurs droits. Parfois elles jouent à saute-mouton...

Mon identité est composite, une sorte de mosaïque "transmise/construite ${ }^{12}$ » et mouvante (comme celle de mes parents d'ailleurs). Je me sens profondément «méditerranéenne », enracinée tour à tour en Suisse et en Amérique du Nord.

Lors de ma première cure analytique, j'ai choisi une analyste française, donc « étrangère » comme je l'étais par rapport à la Suisse. J'ai un souvenir vague (et très lointain dans le temps) de mots surgissant en différentes langues, des mots-corps liés à l'infantile, cherchant une voie d'accès à des ressentis intraduisibles mais je ne saurais en dire plus, le refoulement ayant depuis refait son œuvre. 
Une illustration particulièrement frappante de ce pétrissage corps/langue/psyché est donné par Anne Michaels (1988, 119):

À table [...] il m'enseignait les subtilités de la langue anglaise qui était devenue ma nourriture. Je la mâchais, l'avalais, tâchais de la digérer. À chaque bouchée, un bienêtre mêlé de panique emplissait mon estomac puis irradiait aussitôt dans tout mon corps. [...] pendant que je mastiquais et que j'avalais, avec mes légumes et ma viande, les vocables nouveaux. (Michaels, 1998, 119)

\section{Multilinguisme et profession}

Bien des années plus tard, lors de ma formation à l'Institut Psychanalytique de Montréal, j'ai fait partie d'un séminaire sur la langue maternelle, animé par Bernadette Tanguay. J’ai réalisé que le multilinguisme, familier pour moi, n’en était pas moins sujet d'inquiétante étrangeté et de questionnement.

Dans le registre des psychologues, les langues que je connais sont annoncées. Certains patients arabophones me choisissent tout en ignorant quel dialecte arabe ${ }^{13}$ je maîtrise. Ils ignorent également de quelle religion je suis. Ils présupposent cependant que je suis sensible à leur contexte culturel. J'ai pris l'habitude de dire lors des premières séances de mise en place du cadre et de la consigne d'association libre «Dites ce qui vous vient et dans la langue où ça vous vient ». J'ai emprunté cette formulation à Luisa de Urtebey (1987) ${ }^{14}$.

Au fil des années, je suis devenue attentive aux associations qui surgissent parfois en moi dans une autre langue que le français. En y réfléchissant après-coup, il s'agit le plus souvent d'une irruption de l'infantile - surnoms « codés » donnés à différentes parties du corps, chansons enfantines, proverbes et adages cités par une grand-mère ou une marraine, titres de livres de la Bibliothèque Rose, etc.

Parfois aussi, un mot «étranger » émerge isolément dans une langue ignorée par le patient lui-même, une sorte de «mot écran » qui peut, au cours du traitement, être auréolé par un halo d'associations, reliées à des expériences infantiles significatives et oubliées.

Voici quelques illustrations personnelles, toutes issues de thérapies en face à face.

Ahmed, un patient d'Afrique du Nord s'exprimant parfaitement en français, évoque en berbère (que je ne comprends pas) les sentiments violents d'amour et de haine qui l'animent lorsqu'il me parle de sa mère et en arabe les propos de son père. Chez lui, on parlait les trois langues plus ou moins indistinctement. Pour le moment, je comprends ces changements de langue comme un désir de maintenir, fantasmatiquement, père et mère séparés, tout en ayant lui un lien privilégié avec chacun d'eux. Le rapprochement avec moi se traduira par l'utilisation furtive d'un tutoiement vite réprimé.

La question du choix de l'analyste selon qu'il partage ou pas la langue maternelle du patient se pose. Quelle est la signification du désir que l'analyste parle notre langue maternelle? Désir de fusion? Illusion de communication totale? En fait pour qu'il y ait une communication authentique entre celui qui parle et celui qui écoute, un écart, une différence doit se créer, quelle que soit la langue. 
Myriam, disait «oublier l'Arabe » après avoir passé trois mois de vacances d'été en Europe lorsqu'elle était petite. De retour au Moyen-Orient, elle le récupérait rapidement. Elle se souvint aussi que sa grand-mère maternelle européenne haïssait cordialement le conjoint oriental de sa fille, et ne se gênait pas pour en parler de façon méprisante devant sa petite-fille. Myriam prend conscience, dans l'espace thérapeutique, que le terme "oublier l'Arabe", s'adressait non seulement à la langue mais aussi au compagnon de sa mère...

Tess, femme artiste dans la force de l'âge, de langue maternelle anglaise, maîtrisant bien français et espagnol, consulte parce qu'elle vit une relation amoureuse difficile avec un homme plus âgé, souffrant d'impuissance depuis une opération de la prostate. Elle avait déjà consulté, il y a plusieurs années, parce qu'elle se sentait bloquée dans son processus de création et cela l'avait beaucoup aidée. Je la reçois, en face à face, une fois par semaine, les séances se déroulent en anglais. Je la sens très attentive à ce qui se passe dans son monde intérieur. Cependant lorsqu'elle me parle de sa relation amoureuse je perçois une tristesse et un désarroi qu'elle n'exprime pas et dont elle se défend par des rationalisations. Lors d'une séance, alors qu'elle évoque un souvenir d'enfance (des jeux à coloration sexuelle avec un petit Espagnol), je m'entends lui dire: «El pajarito esta infermo ${ }^{15}$.» Des larmes jaillissent et elle s'exclame: «Now I know... I am grieving a beautiful, potent $\operatorname{cock}^{16}$. »

Je fus aussi surprise de mon intervention intempestive en espagnol que par le jaillissement de son affect et la crudité inhabituelle de son langage. Aucune de nous deux n'a fait de commentaire quant au changement de langue.

Après coup je me suis dit que j'avais dû traduire en espagnol mon association entre les jeux sexuels enfantins et le petit oiseau-pénis puisque j'avais appris cette langue étant adulte mais je n'en ai aucun souvenir conscient.

Bella, jeune femme de père méditerranéen dont j'ai parlé ailleurs (PongisKhandjian, 1998 et 2003) consulte pour se désengluer de la dépression qui l'accable depuis le décès de sa mère. Dans les premiers temps de la thérapie, elle parlait peu de son père, semblait même y être indifférente. De temps à autre, cependant, elle utilisait une expression paternelle, fredonnait un bout de chanson qui, à mon insu, me faisait vibrer. Un souvenir précis m'est resté : Bella évoque une très vieille chanson $I l$ vecchio frack ${ }^{17}$ que son père aimait particulièrement et que je connaissais par cœur. Les dernières lignes de la chanson disent:

Adieu, Adieu, Adieu

Addio al mondo

Ai ricordi del passato

Ad un sogno mai sognato

Ad un attimo d'amore

Che mai più ritornera ${ }^{18}$

Je répète simplement et dans la langue d'origine « un rêve jamais rêvé, un instant d'amour qui ne reviendra plus jamais ». Rêveuse, Bella murmure "Au fond, je 
l'aimais bien mon papa ». Elle se souvient que, petite, à l'entrée à l'école, au travers du regard des autres, elle réalisa qu'elle n'était pas une "pure laine». Elle portait un nom d'assonance étrangère, parlait deux langues, avait des lunches «différents". Elle tenait son père responsable pour cette différence. Mais celui qu'elle pensait ne pas aimer et à qui elle en voulait, c'était le père de l'adolescence, celui qui brandissait sa culture méditerranéenne pour lui imposer un mode vestimentaire, lui interdire de sortir, de fréquenter des garçons. Peu à peu, elle s'est permis d'explorer sa double appartenance, son « roman culturel», s'approprier ce que son père lui avait légué.

Selon Lagache (1956), lorsque l'analysant se met à utiliser occasionnellement une langue étrangère qu'il suppose, à tort ou à raison, ignorée par l'analyste, cela pourrait prendre le sens d'empêcher l'analyste de comprendre, soit pour se faire valoir, soit pour embarrasser ou humilier l'analyste. Si l'analyste connaît cette langue étrangère, il serait préférable, pour Lagache, qu'il renonce à répondre dans cette langue (comme pour montrer au patient qu'il n'a pas été pris au dépourvu et que sa puissance n'est pas en défaut).

Avec Bella, entendre son thérapeute répéter ses mots dans la langue paternelle semble avoir déverrouillé un pan de la mémoire.

Parfois la réapparition de la langue maternelle ne peut être interprétée comme une résistance, il s'agirait plutôt d'un retour aux sources, une demande à l'analyste de jouer le rôle d'une mère (ou d'un père?).

Lagache souligne aussi très finement le sens que peut prendre la convention établie par une patiente: ayant choisi de s'exprimer en anglais et s'attendant que son analyste lui réponde en français, elle était troublée lorsqu'il lui arrivait de parler anglais. Il s'avéra qu'elle demandait à l'analyste de parler français pour éviter la qualité érotique qu'avait pour elle l'accent français en anglais.

Le cas le plus dramatique d'exil linguistique est illustré pour moi par Yue. Orpheline, d'origine chinoise, vivant dans un autre pays asiatique, non scolarisée, elle est parquée vers l'âge de 10 ans avec son frère aîné dans un camp Khmer. Elle y crève littéralement de faim et sa survie est constamment menacée. Â l'adolescence, accueillie comme réfugiée au Canada, sans aucun papier d'identité, un nom et un âge lui sont attribués. Dans la vingtaine, elle apprendra à lire et à écrire, cahin-caha, en français puis en anglais, mais la maîtrise de ces deux langues sera difficile. Chinois et Khmer, intimement liés au contexte polytraumatique, s'effacent peu à peu. La langue maternelle était devenue terre étrangère. «Je n’ai pas de langue maternelle. Je parle mal toutes les langues. Pour les blancs, je suis une asiatique, pour les asiatiques, je suis un phénomène bizarre. Je ne sais pas trop qui je suis", s'écrie-t-elle douloureusement. Ce qui ne l'empêchera pas d'apprendre le langage de la musique, langue universelle par excellence et d'avoir un monde fantasmatique foisonnant.

\section{Conclusion}

Comment conclure un sujet aux multiples ramifications, qui pose plus de questions qu'il n'amène de réponses? 
Une fois de plus, un écrivain - poète cernera l'âme humaine avec ses mots, à la fois ancre et grande voile.

"Je n'aime pas le mot "racines", et l'image encore moins. Les racines s'enfouissent dans le sol, se contorsionnent dans la boue, s'épanouissent dans les ténèbres; elles retiennent l'arbre captif dès la naissance, et le nourrissent au prix d'un chantage: "Tu te libères, tu meurs!" Les arbres doivent se résigner, ils ont besoin de leurs racines; les hommes pas. [...] Pour nous, seules importent les routes. [...] Elles nous promettent, elles nous portent, nous poussent, puis nous abandonnent. Alors nous crevons, comme nous étions nés, au bord d'une route que nous n'avions pas choisie. À l'opposé des arbres, les routes n'émergent pas du sol au hasard des semences. Comme nous, elles ont une origine. Origine illusoire, puisqu'une route n'a jamais de véritable commencement ; [...] Origine insaisissable, puisqu'à chaque croisement se sont rejointes d'autres routes, qui venaient d'autres origines » (Maalouf, 2004).

Je résumerai cet extrait d'Origines, de l'écrivain franco-libanais Amin Maalouf en disant que l'homme n'a pas tant besoin de racines que de chemins, et les langues sont des avenues qui ouvrent des horizons infinis.

\author{
Marie-Ange Pongis-Khandjian \\ 1180, av. Murray \\ Québec G1S 3B6
}

\title{
Notes
}

1. Citation trouvée dans un biscuit chinois!

2. En Italien l'adage «Traduttore, traditore» signifie qu'on trahit toujours la langue d'origine en la traduisant. Le français et l'espagnol l'ont calqué par «Traduction trahison» et «traductor trahidor».

3. Voici quelques psychanalystes issus de ce genre d'environnement: Guy Rosolato, né à Istamboul, en Turquie, a vécu au Liban avant d'émigrer en France. André Green, Piera Aulagnier née Spairani, Jacques Assoun ont tous trois passé les dix-huit premières années de leur vie en Égypte. Moustapha Safouan et Mahmoud Sami Ali ont dû quitter ce pays pour pouvoir continuer à exercer la psychanalyse en France. Fethi Benslama, Tunisien, Mélik Chebel, Algérien, et Adnan Houbballah, Marocain, réfléchissent tous trois en arabe et en français sur les liens possibles entre psychanalyse et islam.

4. Intéressante hypothèse avancée par Brigitte Galtier (1994).

5. Magnifiquement résumé et commenté par Madeleine Vitré (1996) dans un volume de Filigrane, on peut y suivre le déroulement dans le temps de l'intérêt de la psychanalyse pour le polyglottisme.

6. Est multi ou polylingue quelqu'un qui apprend à parler plusieurs langues simultanément.

7. Est polyglotte, quelqu' un qui, une fois la langue maternelle bien établie, en apprend une autre.

8. C'est moi qui souligne en italiques.

9. Romancier d'origine italienne, né en Argentine, il a vécu en Italie, en Espagne puis en France où il fut reçu à l'Académie Française.

10. De parents juifs allemands émigrés en Argentine, Léon et Rebeca Grinberg ont à leur tour émigré en Espagne.

11. L'État d'Israël pour les juifs par exemple.

12. Analogue à l'objet «trouvé/crée» de Winnicott.

13. L'arabe littéraire, appris à l'école est le même dans tous les pays arabes mais chaque pays a un ou deux dialectes différents qui n'est pas nécessairement intelligible par les habitants des autres pays.

14. L. de Urtebey, Dites tout ce qui vous passe par la tête, tout comme cela vous vient et dans la langue où cela vous vient, Revue française de psychanalyse, 2/1988. 
15. «Le petit oiseau est malade». Dans le langage enfantin espagnol le mot pajarito désigne aussi le pénis. C'est aussi le cas en arabe, en français, en grec et probablement dans d'autres langues.

16. "Maintenant je sais... Je suis en deuil d'un beau et puissant pénis ». Quoique le mot anglais "cock" correspondrait plutôt au mot français plus cru "queue" ou "bite"

17. Le vieux frac, chanté par Domenico Modugno.

18. Adieu, adieu, adieu

Adieu au monde

Aux souvenirs du passé

À un rêve non rêvé

À un instant d'amour

Qui ne reviendra jamais

\section{Références}

ANZIEU, D., 1987, Les enveloppes psychiques, Dunod, coll «Inconscient et cultures», Paris.

AMATI-MEHLER, J., 1993, La langue exilée, Revue française de psychanalyse, 3, 917-925.

AMATI MEHLER, J., ARGENTIERI, S., CANESTRI J., 1994, Le Babel de l'inconscient, Collection Fil rouge, PUF.

AMATI-MEHLER, J., 2003, La migration, la perte et la mémoire, Klincksiek, revue de didactolgie des langues-cultures, 2003/3, 131, 329-342.

CANETTI, E., 1998, Histoire d’une jeunesse ; La langue sauvée, 1905-1921, écrits autobiographiques, Albin Michel.

COLIN-ROTHBERG, D., 1988, Interrogations sur la langue analytique à partir du surgissement de langues étrangères pendant la cure, Bulletin de la Société psychanalytique de Paris, 1988, 13.

DE URTEBEY, L., 1985, Fondamentale psychologie, inévitable polyglottisme, Revue française de psychanalyse, 6.

DE URTEBEY, L., 1988, Dites tout ce qui vous passe par la tête, tout comme cela vous vient et dans la langue où cela vous vient, Revue française de psychanalyse, 2.

DE URTEBEY, L., 1993, Des unilingues, Revue française de psychanalyse, 3.

FERENCZI, S., 1933, Confusion des langues entre les adultes et l'enfant, Euvres complètes, tome IV, 125135 .

GALTIER, B., 1994, La seconde langue, moyen de restaurer le conflit, Psychanalyse à l'université, 19, 67100.

GANTHERET, F., 1996 Traces et chair, Moi, monde, mots, Éditions Gallimard.

GOMEZ MANGO, E., 2009, Un muet dans les langues, Éditions Gallimard.

GRINBERG, L., GRINBERG, R., 1971 Identidad et cambio, Ed. Kargieman, Buenos Aires.

GRINBERG, L., GRINBERG, R., 1986, Psychanalyse du migrant et de l'exilé, Césura Lyon Éditions.

HAGĖGE, C., 2009, Dictionnaire amoureux des langues, Plon/Odile Jacob.

KERNBERG, O., 1994, La Babel de l'inconscient, Collection Fil rouge, PUF.

LAGACHE, D., 1956, Sur le polyglottisme en psychanalyse, Le transfert et autres travaux psychanalytiques, Euvres III (1952-1956), PUF, 1980.

MAALOUF, A., 2004, Origines, Grasset, Paris.

MICHAELS, A., 1998, La mémoire en fuite, Boréal, traduit de l'anglais par Robert Lalonde.

PONGIS-KHANDJIAN, M-A., 1998, Un deuil peut en cacher un autre, Études sur la mort. Rites et rituels, 114.

PONGIS-KHANDJIAN, M-A., 2003, Le main à main du flambeau familial: de la répétition à l'appropriation. Les transmissions culturelles intergénérationnelles, Filigrane, 12, 2.

VITRÉ, M., 1996, À propos d'un livre: La Babel de l'inconscient: langue maternelle, langues étrangères et psychanalyse, Filigrane, 5, 31 à 35. 